\title{
UNDERSTANDING AND MODELING OF GRAIN BOUNDARY PINNING IN INCONEL 718
}

\author{
Andrea Agnoli ${ }^{1,2}$, Marc Bernacki ${ }^{1}$, Roland Logé ${ }^{1}$, Jean-Michel Franchet ${ }^{2}$, Johanne Laigo $^{2}$, Nathalie Bozzolo ${ }^{1}$ \\ ${ }^{1}$ Cemef, 1 rue Claude Daunesse; Sophia Antipolis, 06904, France \\ ${ }^{2}$ Snecma Gennevilliers, 171 boulevard Valmy; Colombes, 92702, France
}

Keywords: Inconel 718, Zener pinning, Numerical simulation, Grain growth

\begin{abstract}
The microstructure stability during $\delta$ sub-solvus annealing was investigated in Inconel 718 alloy. A reference dynamically recrystallized microstructure was produced through thermomechanical processing (torsion). The reference microstructure evolution during annealing was analyzed by EBSD (grain size, intragranular misorientation) and SEM ( $\delta$ phase particles). Results confirm that, in the absence of stored energy, the grain structure is controlled by the $\delta$ phase particles, as predicted by the Zener equation. If the reference microstructure is strained $(\varepsilon<0.1)$ before annealing, then stored energy gradients between grains will induce selective grain growth leading to coarsening. The phenomenon is controlled by the balance of three forces (acting on boundaries migration) having the same order of magnitude: capillarity, stored-energy and pinning forces. All these forces could be modeled in a single framework by the level set method. The first numerical results demonstrate the capability of the method to simulate 2D Zener pinning.
\end{abstract}

\section{Introduction}

The mechanical properties of Inconel 718 are greatly influenced by the microstructure that has to be fine and homogenous to ensure the resistance in service conditions. Hence, the control of microstructure evolution during metal forming is a crucial aspect $[1,2]$. The stability of the microstructure has to be under control during each stage of thermomechanical processing, notably during the annealing stage. In Inconel 718, the presence of $\delta$ phase particles $\left(N i_{3} N b\right)$ is exploited during $\delta$ sub-solvus annealing (i.e. at a temperature below the solvus temperature of particles) to limit grain growth through the Zener pinning effect. Nonetheless, under certain conditions the driving forces for grain boundary migration overcome the pinning force leading to the coarsening of the microstructure. In fact, grain boundary migration is driven by two main factors: the capillarity force (linked to the grain boundary curvature) and the stored energy difference across the grain boundary (linked to the local dislocation density).
The aim of this paper is to investigate the influence of the distribution of $\delta$ phase particles and deformation stored energyon the microstructure stability during $\delta$ sub-solvus annealing.

\section{Experimental part}

Experimental techniques

The stability of a reference Inconel 718 microstructure was investigated during $\delta$ sub-solvus annealing (980$1010^{\circ} \mathrm{C}$ ) for $2 \mathrm{~h}$ in an electrical furnace. The reference microstructure was obtained by applying a standard sub-solvus thermomechanical processing to torsion samples, machined from an Inconel 718 billet. The cycle involved annealing at $980-1010^{\circ} \mathrm{C}$, hot deformation (equivalent strain up to 0.8-1.2) and then water quenching. The chemical composition of the billet is shown in Table 1, while the average grain size was equal to $40 \mu \mathrm{m}$ and the $\delta$ phase surface fraction was less than $1 \%$. The radius of torsion samples was $3 \mathrm{~mm}$ and the gauge length was $15 \mathrm{~mm}$. Longitudinal sections were cut (at radius $=0$ ): as the strain is linearly proportional to the radius, this allowed to analyze on the same section all the strain range from zero (at radius $=0$ ) to the maximum value (at radius $=3 \mathrm{~mm}$ ). Then samples were prepared for SEM (ZEISS SUPRA 40 FEG) and EBSD (Bruker CrystAlign) characterization. The preparation consisted of mechanical grinding, followed by fine polishing and finally vibration polishing for at least 12 hours (using an anti-agglomerating $\mathrm{SiO}_{2}$ colloidal solution).

\begin{tabular}{ccccccc}
\hline $\mathrm{Ni}$ & $\mathrm{Cr}$ & $\mathrm{Fe}$ & $\mathrm{Nb}$ & $\mathrm{Mo}$ & $\mathrm{Al}$ & $\mathrm{Co}$ \\
\hline bal. & 18 & 17.44 & 5.39 & 2.96 & 0.46 & 0.14 \\
\hline \hline $\mathrm{Ti}$ & $\mathrm{Mn}$ & $\mathrm{Si}$ & $\mathrm{Cu}$ & $\mathrm{C}$ & $\mathrm{Ta}$ & $\mathrm{B}$ \\
\hline 0.1 & 0.9 & 0.08 & 0.03 & 0.023 & 0.01 & 0.0041 \\
\hline
\end{tabular}

Table 1: Chemical composition of the Inconel 718 billet (weight percent)

The fraction and morphology of $\delta$ phase particles were determined by image analysis using the UTHSCSA Image Tool software. At least 5 BSE (back-scattered elec- 
trons) contrast images per sample were analyzed: each image corresponds to an area of $100 \times 150 \mu m$. Grain size and intragranular misorientation were determined by analysis of EBSD raw data sets using the TSL OIM Analysis software. EBSD measurements were performed choosing an appropriate step size $(0.2-1 \mu \mathrm{m})$ for each microstructure scale. For the evaluation of grain size the tolerance angle was set to $5^{\circ}$ and twins (defined by a misorientation along the axis $<111>$ equal to $60^{\circ}$ with a tolerance of $5^{\circ}$ ) were ignored. Intragranular misorientation was estimated either by calculating the GOS (Grain Orientation Spread) or the GAM (Grain Average Misorientation) parameter provided by the OIM software. The GOS is the average misorientation angle between each measuring point (in a grain) and the average grain orientation. Thus, it does not depend on the step size and it takes into account long-range orientation gradients. The GAM is the average misorientation angle between each point (in a grain) and its neighbors. It depends on the step size as it takes into account only point-to-point orientation gradients: therefore, when using this parameter the step size must be also specified.

\section{Experimental results}

Reference microstructure The reference microstructure is almost fully $(90 \%)$ recrystallized. Figure 1 shows that the microstructure is equiaxed and the average grain size is about $10 \mu \mathrm{m}$. Nonetheless it is possible to note the presence of few bigger grains $(30-40 \mu \mathrm{m})$ which did not recrystallize. The average $\delta$ phase surface fraction (that coincides with the volume fraction if a sufficient number of measures is considered) was found to be in the range of $1.7 \pm 0.3 \%$. It is to note that a strict control on the fraction of $\delta$ phase, especially at low particles densities, was quite hard to be achieved as chemical composition heterogeneity in Inconel 718 always affects the density of particles. Figure 2 shows that the distribution and morphology of the particles is quite heterogeneous. Notably, if the distribution of the minor axis (or width) of particles is fairly centered around the average value $(0.3 \mu \mathrm{m})$, on the contrary the distribution of the major axis (length) is wider and spans up to $3 \mu \mathrm{m}$.

Intragranular misorientation was evaluated through the GOS parameter. This parameter provides relative information about the hardening state of single grains, as the average intragranular misorientation of a grain can be linked to the density of dislocations (which accomodate the crystal deformation). Figure 3 shows the GOS distribution is centered at about $0.5^{\circ}$, a value which is close to the EBSD system angular resolution. As the distribution is also very narrow, it can be considered that roughly $90 \%$ are strain-free recrystallized

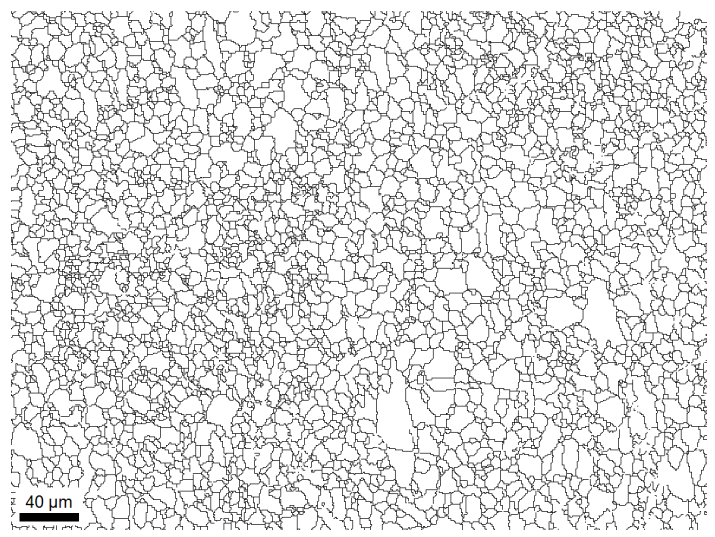

(a)

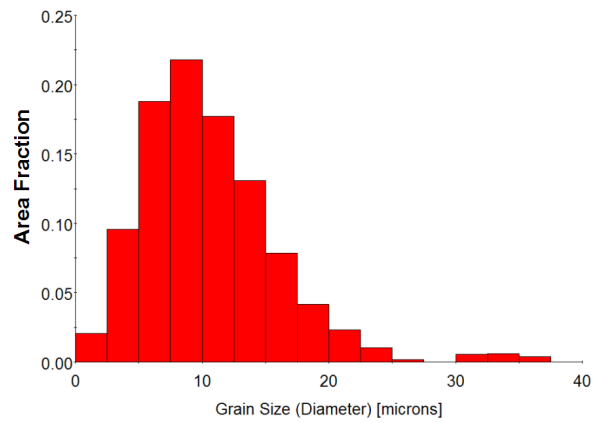

(b)

Figure 1: Grain size distribution in the reference microstructure: (a) grain boundaries in black (twins excluded), (b) grain surface fraction

grains. The rest of the grains are more or less hardened: in particular, grains with GOS higher than $3^{\circ}$ are clearly unrecrystallized hardened grains.

$\delta$ sub-solvus annealing A sample of the reference microstructure was $\delta$ sub-solvus annealed $\left(980-1010^{\circ} \mathrm{C}\right)$ for $2 \mathrm{~h}$ to investigate the stability of the microstructure. The comparison of Figure 1 and 4 shows that there was little or no grain growth despite the high temperature and prolonged annealing time. In fact, as it is well-known in literature and in the industrial field, the presence of $\delta$ phase particles in Inconel 718 can hinder grain growth. This phenomenon is known as Zener pinning and the relation between the stable grain size and particles can be described by the following simple equation [3]:

$$
<D>=\alpha \frac{4}{3} \frac{<r>}{<f>},
$$

where $\langle D\rangle$ corresponds to the average diameter of (randomnly distributed spherical) grains, $\langle r\rangle$ and $<f>$ are respectively the average radius and volume 


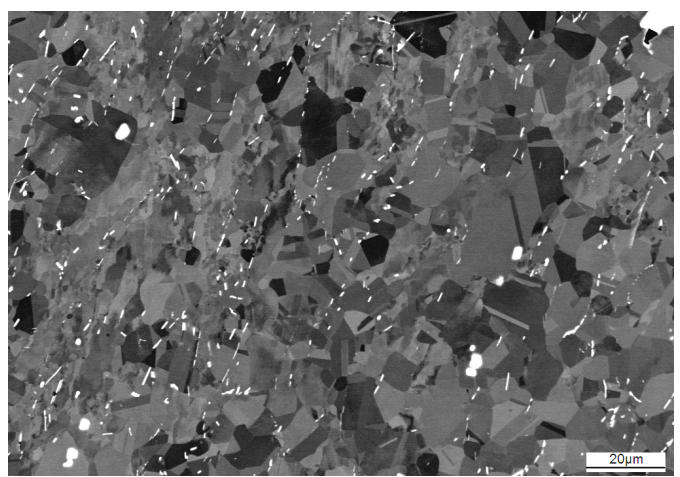

(a)

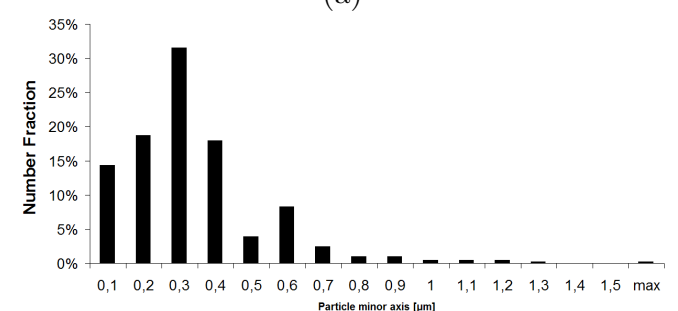

(b)

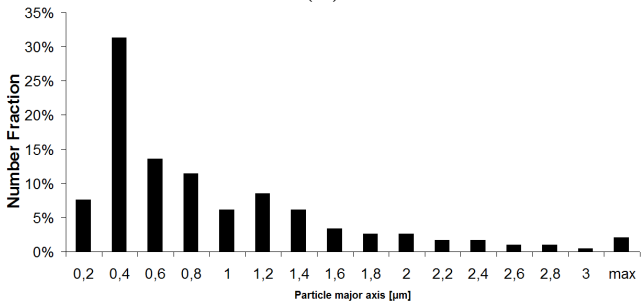

(c)

Figure 2: Distribution of the $\delta$ phase in the reference microstructure: (a) BSE image showing the $\delta$ phase as small white particles and few carbides $(\mathrm{NbC})$ as bigger particles, (b) minor axis and (c) major axis particle number fraction

fraction of (spherical) secondary-phase particles and $\alpha$ is a constant $(0.25<\alpha<0.5)$. The value of the parameter $\alpha$ is influenced by the microstructure properties (boundaries energies and average curvature, particle distribution and shape, etc,). As shown in Figure 2, the $\delta$ phase morphology is quite complex and particles can be hardly considered as spheres. Nonetheless, a solution is to evaluate the equivalent radius of spherical particles having the same volume as experimental particles. Then, it is possible to plot Eq.(1) as a function of particle fraction. Figure 5 shows that the experimental data obtained by annealing the reference microstructure (ref) falls in between the two curves. Then, despite the simplicity and all the assumptions behind Eq.(1), it still allows to understand and describe why significant grain

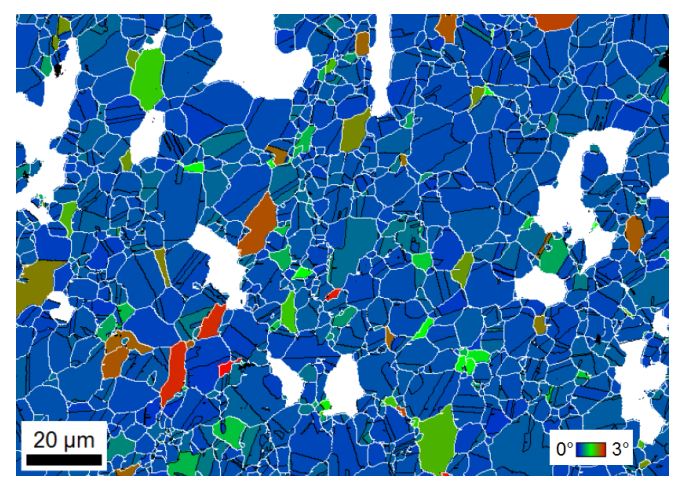

(a)

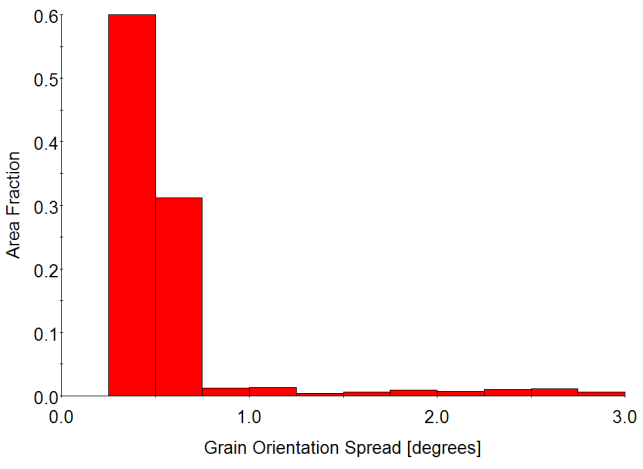

(b)

Figure 3: Grain orientation spread (GOS) distribution in the reference microstructure: (a) grain boundaries in white, twins in black (white grains have higher GOS values than $3^{\circ}$ ), (b) GOS surface fraction

growth did not occur after annealing. More precisely, the analysis of the microstructure by SEM suggests that some grains indeed grew over passing the Zener limit, but only in regions where the local fraction of particles was much lower compared to the surroundings. A support for this explanation is given in Figure 6, which also shows that after annealing the average $\delta$ phase fraction is higher (about $4 \pm 0.5 \%$ ) compared to the initial state (about $1.7 \pm 0.3 \%$ ) shown in Figure 2. In fact, during $\delta$ sub-solvus annealing precipitation (mostly at grain boundaries) and particle growth occur.

$\delta$ sub-solvus annealing after low strain A sample of the reference microstructure was strained by hot torsion $\left(980-1010^{\circ} \mathrm{C}\right.$ ) reaching an equivalent strain of 0.1 (at radius $=3 \mathrm{~mm}$ ) and then it was water quenched to room temperature to be characterized. Finally the sample was $\delta$ sub-solvus annealed for $2 \mathrm{~h}$ to investigate the influence of previous straining on the stability of the microstructure. As expected, the microstructure of the strained sample after quenching does not differ much 


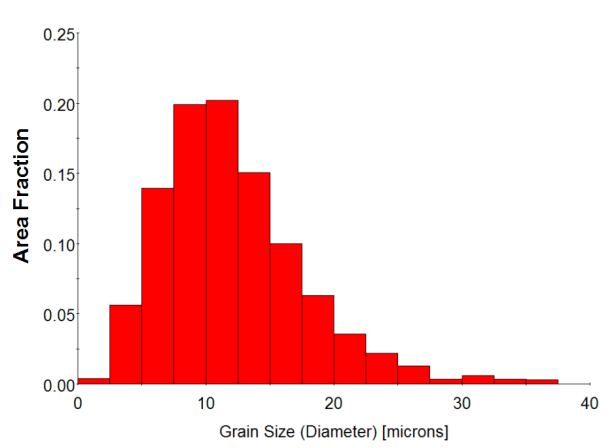

Figure 4: Grain size distribution (surface fraction) in the reference microstructure after $\delta$ sub-solvus annealing for 2 hours

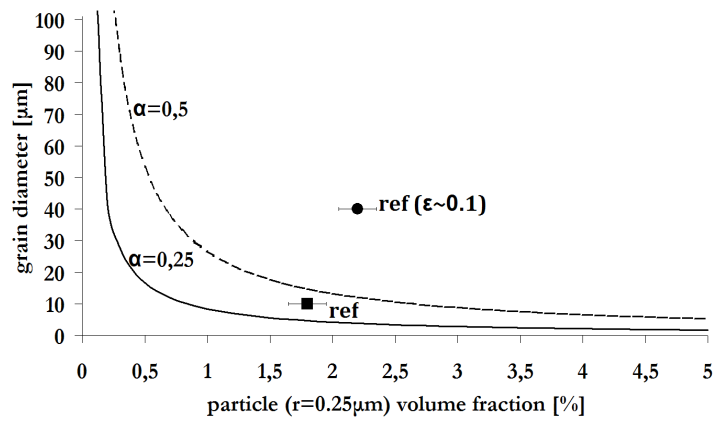

Figure 5: Comparison of grain size predicted by Eq.(1) and values measured on reference (ref) and deformed $(\operatorname{ref}(\varepsilon=0.1))$ samples after $\delta$ sub-solvus annealing for 2 hours

from the reference microstructure in terms of grain size and $\delta$ phase distribution. More precisely, it was found an average grain size of $10 \mu \mathrm{m}$, an equivalent morphology of particles and only a slightly higher $\delta$ phase fraction $(2.2 \pm 0.3 \%)$. On the contrary, the average intragranular misorientation in the strained sample is higher and it increases with strain. Figure 7 shows that as strain increases the distribution widens by shifting towards higher values.

After annealing of the strained sample, the grain size greatly increases as shown in Figure 8. Moreover, the final microstructure appears to be linked to the degree of the local strain. In the lower region where the strain is close to 0.07 , there are two populations of fine initial grains $(10 \mu \mathrm{m})$ and overgrown grains of size up to $100 \mu \mathrm{m}$. In the upper region where the strain is close 0.1 , the microstructure is more homogeneous, composed mostly by one overgrown grain population (marked by a red box) with an average grain size of $40 \mu \mathrm{m}$. Overgrown grains are characterized by very low GOS values, while

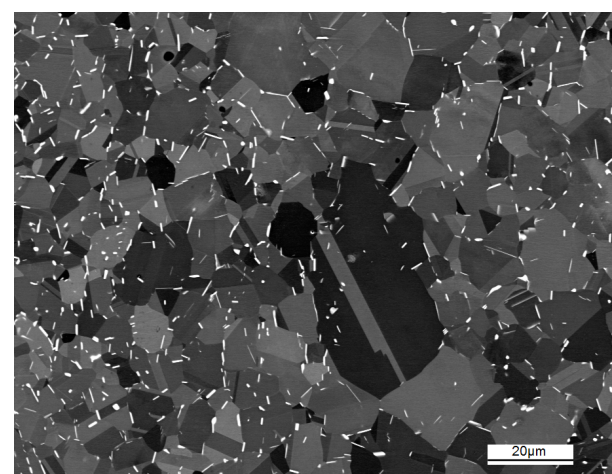

(a)

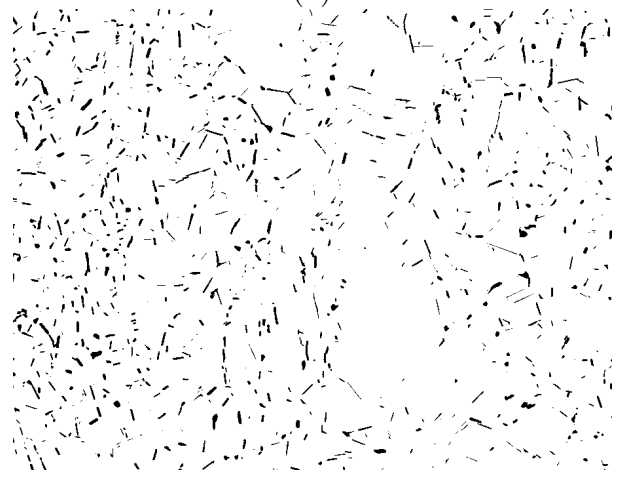

(b)

Figure 6: Grain bigger than the Zener limit in the reference microstructure after $\delta$ sub-solvus annealing for 2 hours: (a) BSE image, (b) $\delta$ phase filtered

fine grains have higher GOS values as a function of the strain degree (see Figure 7).

Such microstructure evolution cannot be explained as a case of normal grain growth controlled by Zener pinning: as it is shown in Figure 5, the final grain size in the strained sample $(\operatorname{ref}(\varepsilon=0.1)$ is not related to the local fraction of particles. Neither recrystallization appears to be involved as the applied strain at hot temperature $(\varepsilon \leq 0.1)$ does not seem to be enough to trigger the nucleation of new grains. Abnormal grain growth may be involved in this case as it occurs when normal grain growth is inhibited (by the presence of particles) and/or certain grains enjoy some growing advantage over their neighbors, as bigger size (due to broad initial grain size distributions), higher boundary mobility (due to texture) or lower boundary energy (due to "special" grain boundaries) [3].

These three possible origins of the phenomenon were tested but no evidence was found for any of them: details will be presented in a forthcoming publication. On the contrary, the microstructure evolution in the strained sample appears to be a case of strain- 


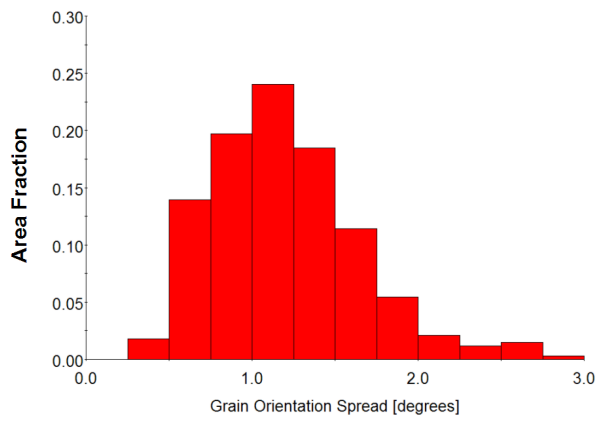

(a)

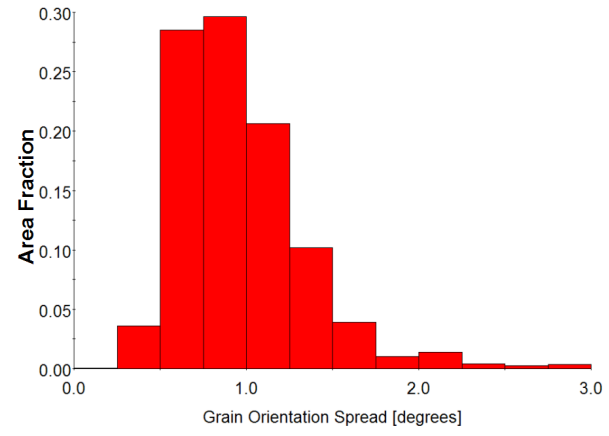

(b)

Figure 7: Grain orientation spread (GOS) distribution (surface fraction) in the strained sample after quenching as a function of equivalent strain: (a) 0.1 and (b) 0.07

induced abnormal growth [4]. This phenomenon leads to inhomogeneous grain growth after annealing of critically strained materials, where low dislocation density microstructures are formed and then few strain-free (low stored energy) grains can grow at the expanse of strained grains (high stored energy) during annealing. If the GOS parameter can describe qualitatively the energy stored in grains, then Figure 7 shows that gradients of stored energy are present in the initial microstructure. In addition, Figure 8 shows that during annealing few low energy grains grew selectively at the expense of higher energy grains pinned by particles. Since the number of growing grains is expected to increase as the width of the distribution of stored energies is higher, the comparison of the GOS histograms of Figure 7 can explain the grain size dependance on strain. The plausibility of this scenario can be quantitative assessed by estimating the three driving forces which govern grain boundary migration: the capillarity force, the stored energy difference across the grain boundary and the Zener pinning force. If the boundary migrates driven by the capillarity effect, then the driving pressure can be ex-

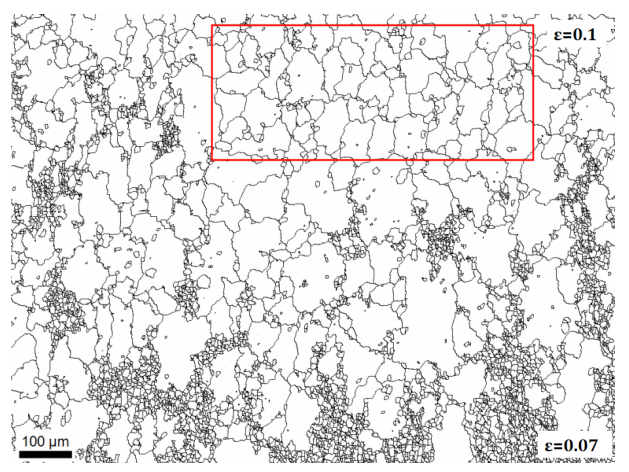

(a)

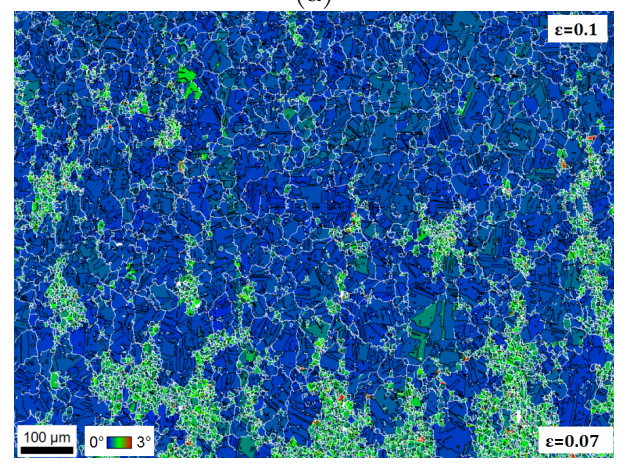

(b)

Figure 8: Microstructure of strained sample $(0.07 \leq \varepsilon \leq$ 0.1 ) after annealing: (a) grain boundaries in black (population of $40 \mu \mathrm{m}$ grains marked by a red box), (b) grain orientation spread (GOS) map with grain boundaries in white and twins in black

pressed as [3]:

$$
P_{G}=\gamma<k>
$$

where $\gamma$ is the grain boundary energy and $k$ is its mean curvature. In the case of spherical grains of radius $\mathrm{R}$, $<k>=\frac{2}{R}$ and so $P_{G}=\frac{2 \gamma}{R}$. However, in real microstructures grains have different shapes and the effective driving force is found to be much lower as compared to the case of spherical particles. Based upon experimental observations on different (pure) materials, the relation between the mean grain size radius $(\mathrm{R})$ and the effective driving force can be estimated as [5]:

$$
P_{G}=\frac{\gamma}{4 R}
$$

If the grain boundary separates two grains of different stored energy, then the driving pressure is equal to the difference of the energies: $P_{R}=\triangle E$. The energy is linked to the dislocation density $\rho$ by this equation [3]:

$$
E=0.5 \rho G b^{2},
$$


where $G$ is the shear modulus and $b$ is the Burgers vector. The density of geometrically necessary dislocations in a grain is linked to intragranular misorientation. If it is assumed that the torsion of a cylinder requires a series of twist subgrain boundaries, each containing two perpendicular arrays of screw dislocations, then the density can be estimated to a first approximation by EBSD analysis as [6]:

$$
\rho=\frac{2}{b} \frac{<\theta>}{\triangle x}
$$

where $\langle\Delta \theta>$ is the mean misorientation angle between neighbor pixels in a grain and $\triangle x$ is the measuring step. The Grain average misorientation (GAM) is defined as $\frac{\langle\Delta \theta\rangle}{\Delta x}$. The presence of secondary-phase particles of radius $(r)$ and volume fraction $(f)$ hinders boundary migration with a pinning pressure [3]:

$$
P_{P}=\frac{3 \gamma f}{2 r} .
$$

Table 2 shows the comparison of driving forces and pinning force estimated in the strained $(\varepsilon=0.1) \mathrm{mi}-$ crostructure before annealing. It is to note that the stored energy pressure has been calculated for a boundary between two grains where the difference of $\langle\Delta \theta\rangle$ is $0.5^{\circ}$. This value has been chosen by considering the distribution width of the grain average misorientation (GAM) in the strained sample $(\varepsilon \leq 0.1)$ after quenching (Figure 9). It is to remark that pressures magnitudes

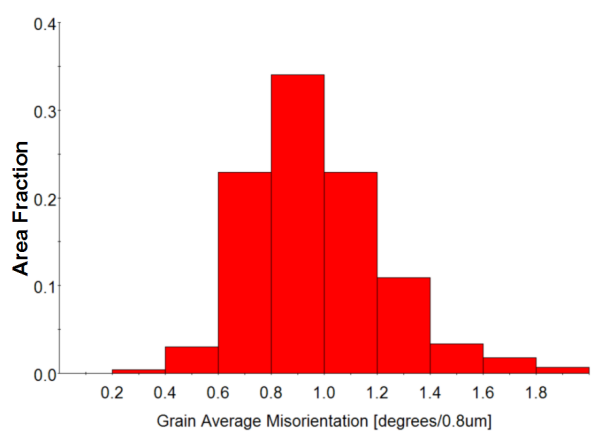

Figure 9: Grain average misorientation (GAM) distribution (surface fraction) in the strained sample $(\varepsilon=0.1)$ after quenching

presented in Table 2 are all of the same order. Even if these values correspond to a rough approximation, they are sufficient to affirm that all phenomena can play an important role in the evolution of the microstructure. However, since the balance of the driving forces varies locally with the microstructure, any analysis of the phenomenon under study based on average properties of the microstructure may not be appropriate. In fact, this result suggests that the understanding of the phenomenon

\begin{tabular}{cc}
\hline Pressure & {$[\mathrm{kPa}]$} \\
\hline Pinning (Eq.(6)) & -80 \\
\hline Stored energy (Eq.(4), $\left.<\triangle \theta>=0.5^{\circ}\right)$ & 120 \\
\hline Capillarity (Eq.(3)) & 30 \\
\hline
\end{tabular}

Table 2: Comparison of approximate driving forces and pinning force in the strained sample $(\varepsilon \leq 0.1)$ after quenching

would require a mesoscopic model able to describe the evolution of the microstructure at the scale of a representative volume element (RVE).

\section{Numerical part}

Grain growth model

Microstructure evolution simulation is based on a level set description of interfaces in a finite-element context . This approach was already used to simulate both $2 \mathrm{D}$ and $3 \mathrm{D}$ primary recrystallization [7] and grain growth [8] in poly-crystals. Anisotropic meshing and remeshing techniques were used to accurately describe interfaces, both for the modelling of plastic deformation using crystal plasticity, and for updating the grain boundary network at the recrystallization stage. A level set function $(\phi)$ is defined over a domain $\Omega$ as a sign distance function (positive inside and negative outside) from an interface $(\Gamma)$. Then, the level 0 of the function allows to determine the interface:

$$
\left\{\begin{array}{l}
\phi(x)=d(x, \Gamma), \quad x \epsilon \Omega \\
\Gamma=\{x \epsilon \Omega, \phi(x)=0\} .
\end{array}\right.
$$

If a microstructure contains $N_{G}$ grains, then one level set function $\left(\phi_{i}\right)$ per grain $G_{i}$ has to be considered. In the case of capillarity driven grain growth (no stored energy), assuming a constant mobility $(M)$ and boundary energy $(\gamma)$, the kinetic law for the grain boundary $\Gamma_{i}$ of the grain $G_{i}$ can be described as:

$$
\vec{v}_{i}=-M \gamma \kappa_{i} \vec{n}_{i},
$$

where $\vec{n}_{i}=\frac{\nabla \phi_{i}}{\left\|\nabla \phi_{i}\right\|}$ and $\kappa_{i}=-\nabla \cdot \vec{n}_{i}$ correspond, respectively, to the unit normal and the mean curvature of $\Gamma_{i}$. Then, microstructure evolution is described by this set of convection equations:

$$
\left\{\begin{array}{l}
\frac{\partial \phi_{i}}{\partial t}-M \gamma \kappa_{i} \overrightarrow{n_{i}} \cdot \nabla \phi_{i}=0 \\
\phi_{i}(t=0, x)=\phi_{i}^{0}(x), \quad \forall i \epsilon\left\{1, \ldots, N_{G}\right\} .
\end{array}\right.
$$

If all level set functions satisfy the metric property $\left\|\nabla \phi_{i}\right\|=1$, then the problem of Eq.(9) can be reformulated as a set of diffusion equations, where the explicit 
calculation of the curvature and the normal of the grain boundaries can be avoided:

$$
\left\{\begin{array}{l}
\frac{\partial \phi_{i}}{\partial t}-M \gamma_{i} \triangle \phi_{i}=0 \\
\phi_{i}(t=0, x)=\phi_{i}^{0}(x), \quad \forall i \epsilon\left\{1, \ldots, N_{G}\right\} .
\end{array}\right.
$$

As the resolution of Eq.(10) can result in values of $\left\|\nabla \phi_{i}\right\|$ different from unity, then it is necessary to reinitialize all level set functions (so that $\left\|\nabla \phi_{i}\right\|=1$ ), by solving this set of reinitialization equations:

$$
\left\{\begin{array}{l}
\frac{\partial \phi_{i}}{\partial t}+\lambda s_{i}\left(\left\|\nabla \phi_{i}\right\|-1\right)=0 \\
\phi_{i}(t=0, x)=\phi_{i}^{0}(x), \quad \forall i \epsilon\left\{1, \ldots, N_{G}\right\} .
\end{array}\right.
$$

Finally the simulation of grain growth involves all these steps at each time increment (details for each step are given in $[7,8,9])$ :

- Grain boundary migration is calculated by solving Eq.(10) for each level set functions.

- All level set functions are modified to remove vacuum regions appearing at multiple grain junctions: $\phi_{i}(x, t)=\frac{1}{2}\left[\left(\phi_{i}(x, t)-\max _{j \neq i}\left(\phi_{j}(x, t)\right)\right]\right.$.

- All level set function are reinitialized (so that $\left.\left\|\nabla \phi_{i}\right\|=1\right)$, by solving Eq.(11), in a narrow zone around all interfaces.

- (Only) negative level set function are removed, as they correspond to disappeared grains.

- Anisotropic remeshing is performed when at least one grain boundary leaves the anisotropically meshed layer (that is a narrow zone of refined mesh around all interfaces).

Zener pinning simulation In literature the pinning effect of secondary-phase particles on grain growth has already been modeled by Monte Carlo, Phase-field and Boundary-tracking methods. Monte Carlo models $[10,11]$, which were the first to be developed, and Phase-field models [12, 13], which have gained more attention in the last years, are able to simulate both the $2 \mathrm{D}$ and 3D Zener pinning phenomenon. On the contrary, Boundary-tracking models are limited to 2D simulations [14] or 3D simulation of the interaction of a single grain boundary with particles $[15,16]$. Overall, while all methods can effectively simulate Zener pinning in the simple case of spherical particles, there is not yet a model that can deal with particles whose shape is more complex than an ellipsoid one. In addition, all models still assume that the surface tension between grains and particles is isotropic. It is to note that these two parameters may have an important role on the stability of the microstructure.
The level-set method, described previously, can model Zener pinning without any restrictive assumption on particle shape or boundary energy isotropy. This derives from the fact that the presence of particles is inherently taken into account via its effect on grain boundary curvature. In practice, the model treats particles as simple voids in the mesh, so the shape of particles is limited only by the complexity of the mesh. Moreover, as described in Figure 10, the particle-grain boundary energy anisotropy, which determines the angle $(\alpha)$ of the grain boundary at the interface with a particle, according to the equation $\sin (\alpha)=\left(\gamma_{2 \mathrm{P}}-\gamma_{1 \mathrm{P}}\right) / \gamma[3]$, can be defined in a very simple way by applying the boundary condition $\nabla \phi_{i} \cdot n=\sin (\alpha)$ (with $n$ the outside normal of the particle) that appears in the weak formulation of the microstructure evolution defined by Eq.(10):

$$
\begin{gathered}
\int_{\Omega} \frac{\partial \phi_{i}}{\partial t} v d \Omega+\int_{\Omega} \gamma M \nabla \phi_{i} \cdot \nabla v d \Omega= \\
\int_{\Gamma} \gamma M \nabla \phi_{i} \cdot \bar{n} v d \Omega
\end{gathered} \quad \forall v \in H^{1}(\Omega) .
$$

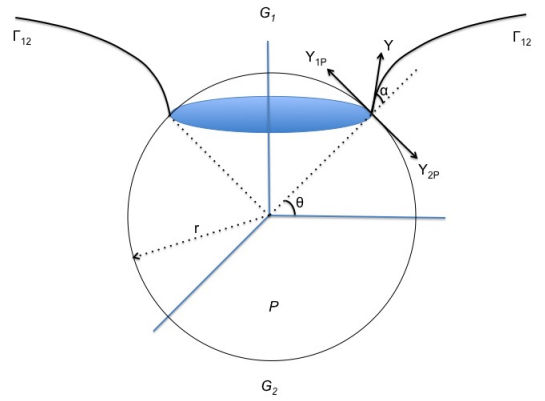

Figure 10: Description of the interaction between a particle $P$ and a grain boundary $\Gamma_{12}$ of boundary energy equal to $\gamma$

For example, if the particle is incoherent, which undermines isotropic boundary energy, it remains to impose a right angle between the particle and the grain boundary and so a null boundary condition in Eq.(12).

Digital microstructure generation

An example of $2 \mathrm{D}$ digital microstructure is given in Figure 12. It is characterized by the initial grain structure, that is defined by level set functions, and by the distribution of particles, which coincides with the voids in the mesh. Concerning the grain generation methodology in a finite element mesh, the coupled "Advancing front packing/Voronoï-Laguerre tesselation" method developed by Hitti [17] in a level-set context is used. The initial grain structure was built in two steps: generation of a dense circle distribution thanks to an optimise advancing front packing algorithm, then calculation of 
level set functions of the Laguerre cells (the grains) from the circles using a Voronoï-Laguerre method. Compared to the standard Voronoï method, which allows to generate microstructures fitting only an average grain size value, in the methodology used, Laguerre cells are generated so that the resulting distribution can fit (in terms of equivalent radius) a grain size distribution measured experimentally. Figure 11 shows that the numerical distribution fits fairly well the experimental one: the error $\left(\frac{\sum\left(f_{E X P} f_{N U M}\right)^{2}}{\sum f_{E X P}^{2}} \%\right)$ is only $3 \%$ for a size domain equal to $[320 \mu \mathrm{m} \times 225 \mu \mathrm{m}]$.

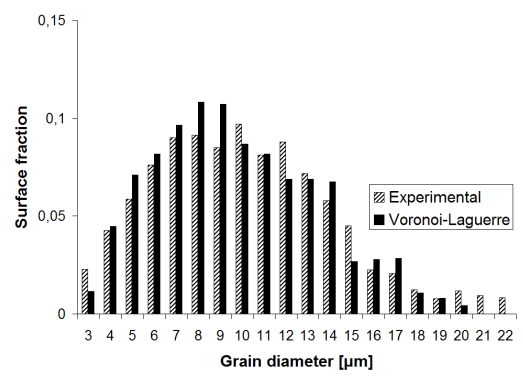

Figure 11: Comparison of the grain size distribution measured experimentally and generated numerically by the Voronoï-Laguerre method ( 1450 grains $)$

The generation of particles first involved the creation of the particles boundary mesh (line elements) fitting a certain particle population (size, distribution). Then this mesh was used to remesh the simulation domain (triangle elements), by removing all elements which were inside the particles boundaries. It is to note that this last operation required the use of anisotropic meshing around particles in order to obtain smooth interfaces [8]. Two types of particles populations were generated. The first type is made of randomly distributed monodisperse spherical particles generated again thanks to the microstructure builder developed in [17]. The second type of population reproduces the same morphology and distribution of particles as they appear on a BSE image (see Figure 13 for a zoom in a [30 $\mathrm{m} \times 20 \mu \mathrm{m}]$ domain). For this purpose, a marching squares method was applied on a binary image of the $\delta$ phase (as shown in Figure 6) to build the boundary mesh of particles necessary to generate the finite element mesh of the simulation domain. Figure 13 shows that in the final mesh the population of particles closely resembles the experimental one. More precisely, only the smallest particles, hardly visible even on the binary image, are not taken into account.

\section{Zener pinning simulation}

The following $2 \mathrm{D}$ simulations were performed using the C++ FE library "CimLib" developed in Cemef.

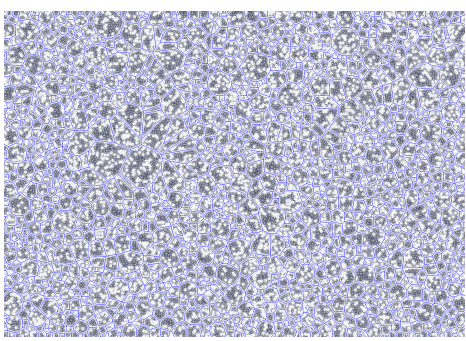

(a)

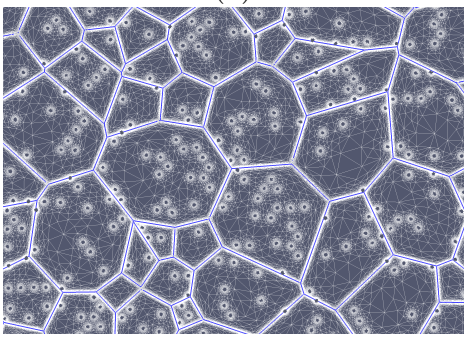

(b)

Figure 12: Example of a digital microstructure in a domain $[320 \mu \mathrm{m} \times 225 \mu \mathrm{m}]$ : average numerical grain size $=8 \mu \mathrm{m}$, particle radius $=0.25 \mu \mathrm{m}$ and surface fraction $=2 \%$. Grain boundaries are shown in blue as the zero level of all level set functions (a). Detail showing particles which are not meshed and the anisotropic mesh layer surrounding grain and particle interfaces (b). Number of mesh element is equal to 5500000 .

The simulation domain was limited to a rectangle of dimensions $[100 \mathrm{x} 100 \mu \mathrm{m}]$ to reduce computation time, but larger domains can be simulated (results will be presented in a forthcoming publication). The average background mesh size was $1 \mu \mathrm{m}$, the thickness of the anisotropic layer was set to $0.25 \mu \mathrm{m}$ and the refined mesh size in the direction perpendicular to grain interfaces was about $0.025 \mu \mathrm{m}$ (see Figure 12).

Simulations of grain growth during $\delta$ sub-solvus annealing of three Inconel 718 microstructures are presented. Each microstructure has the same initial grain population (average grain size $8 \mu \mathrm{m}$ ), as defined in Figure 11. The particle population is different in each microstructure:

1. randomly distributed spherical particles (radius $=0.4 \mu \mathrm{m}$, surface fraction $=1 \%$ )

2. randomly distributed spherical particles (radius $=0.4 \mu \mathrm{m}$, surface fraction $=2 \%$ )

3. experimental distribution of $\delta$ phase particles (equivalent radius $=0.25$, surface fraction $=2 \%$ )

The equivalent Zener equation (see Eq.1) for 2D sys- 


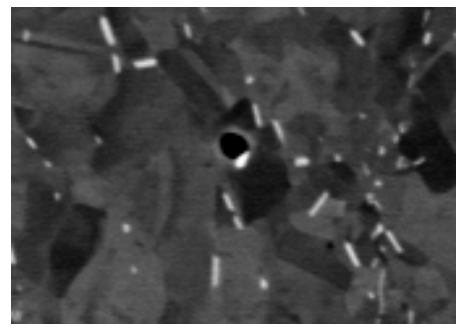

(a)

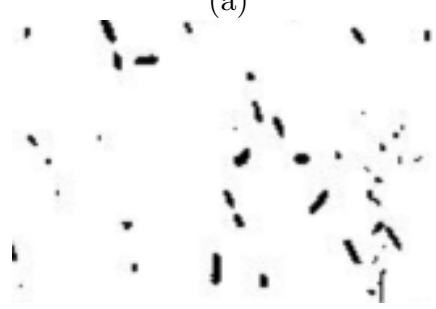

(b)

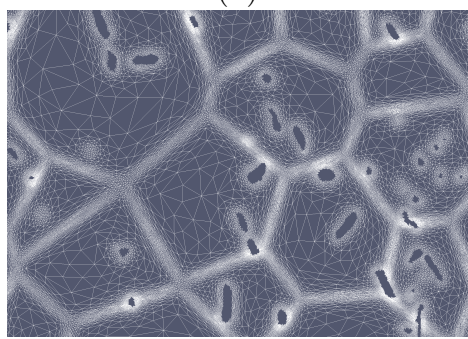

(c)

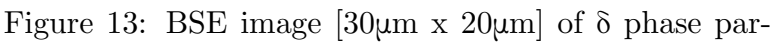
ticles (a) and corresponding binary image (b) used to generate the mesh (c) where particles and grains appear surrounded by anisotropic meshing. Global finite element mesh in the domain [320 $\mu \mathrm{m} \times 225 \mu \mathrm{m}]$ is made of 6500000 elements.

tems, can be expressed as [18]:

$$
<D>=2 \sqrt{3} \frac{<r>}{\sqrt{<f>}} .
$$

In Figure 14 Eq.(13) is plotted along with pinned grain sizes as obtained by numerical simulations. The plot shows that numerical results are close to the theoretical curve, but they overestimate the size of grains. This could be simply due to the fact that the size of the domain is small, then the number of grains is statistically limited and the influence of the domain boundaries can not be neglected. Another reason could be linked to the initial grain size $(8 \mu \mathrm{m})$ imposed in all simulations, which is already close to the pinned grain size, and the initial distribution of particles, which is uncorrelated to grain boundaries. As a consequence, grains continue to grow overpassing the theoretical limit until they interact with enough particles. Similar results, concerning the influence of the initial microstructure on 2D Zener pinning simulation were found in [18]. In fact, Figure 14 shows that the deviation from the theoretical curve is the smallest when the pinned theoretical grain size is the highest (simulation with $1 \%$ particles). The evolu-

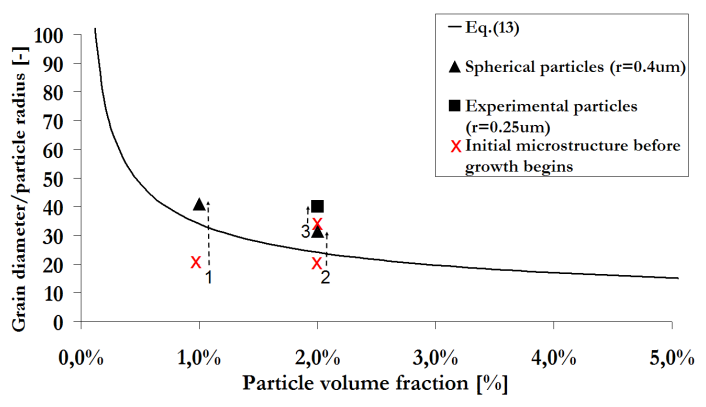

Figure 14: The final grain size (normalized by the particle radius) when grain growth stops (black square and triangles) is compared with the predicted final grain size of Eq.(13). Arrows underline how much the final microstructure has evolved from each different initial microstructure (red crosses).

tion of average grain size with time is shown in Figure 15 for the case of spherical particles. The plot shows that grain growth is fast during the first 100 seconds, where the fraction of particles on grain boundaries is still low. As time increases, the fraction of pinned grain boundaries increases and a stable grain size is reached after 600-700 seconds. In the microstructure with experimental particles, grain growth stops earlier, after 50-100 seconds. In fact, despite having the same surface fraction of particles $(2 \%)$, in this case the number of particles is higher and so the total pinning pressure is stronger. Figure 16 shows that the evolution of the microstructure with experimental particles reaches a stable state after only 50s. Then, the experimental distribution of particles alone can not explain the phenomenon of selective grain growth in torsion tests. That is, the phenomenon can be reproduced numerically only if gradients of stored energy can be added to the simulation of Zener pinning taking into account real particles distributions.

\section{Conclusions}

Experimental results show that a typical recrystallized (no stored energy) microstructure is stable during annealing. In this case grain growth is controlled by the presence of $\delta$ phase particles as predicted by the Zener equation. On the contrary, if stored energy gradients between grains are introduced in the microstructure, then the Zener limit can be passed over and this leads to the 


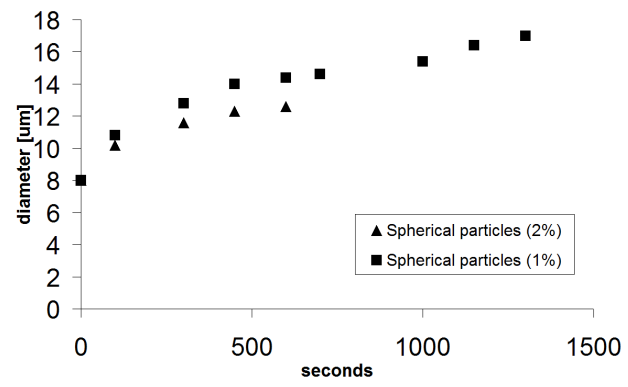

Figure 15: Evolution of average grain size during growth as a function particles fraction

selective growth of few grains.

Numerical results show that 2D Zener pinning can be simulated by the level-set method, taking into account the real morphology and distribution of $\delta$ phase particles. Future work will involve the simulation of 3D Zener pinning, taking into account also the real topology of grains. Moreover, the addition of gradients of stored energy will enable to simulate the observed case of selective grain growth.

\section{References}

[1] J. Thomas, E. Bauchet, C. Dumont, F. Montheillet in Superalloys 2004, pp. 959-968.

[2] B. Flageolet, O. Yousfi, Y. Dahan, P. Villechaise, J. Cormier in 7th International Symposium on superalloy 718 and derivatives, pp. 595-606.

[3] F. Humphreys, M. Hatherly, Recrystallization and related annealing phenomena, 2004.

[4] T. A. Bennett, P. N. Kalu, A. D. Rollett, Microsc.Microanal., 2011, 17, 1-6.

[5] P. A. Manohar, M. Ferry, T. Chandra, ISIJ International, 1998, 38, 913-924.

[6] M. Calcagnotto, D. Ponge, E. Demir, D. Raabe, Materials Science and Engineering: A, 2010, 527, $2738-2746$.

[7] M. Bernacki, H. Resk, T. Coupez, R. E. Logé, Modelling and Simulation in Materials Science and Engineering, 2009, 17, 064006.

[8] M. Bernacki, R. Logé, T. Coupez, Scripta Materialia, 2011, 64, 525-528.

[9] M. Bernacki, Y. Chastel, T. Coupez, R. Logé, Scripta Materialia, 2008, 58, 1129-1132.

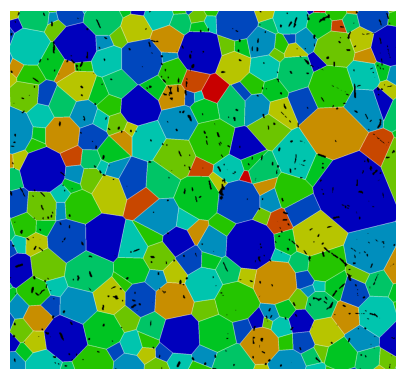

(a)

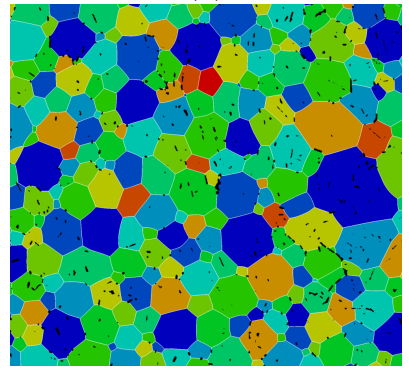

(b)

Figure 16: Evolution during annealing of the microstructure with experimental particles in a domain $[100 \mu \mathrm{mx} 100 \mu \mathrm{m}]$. Grain boundaries are in white and colors are used to identify grains. Initial microstructure (a): average numerical grain size $=8 \mu \mathrm{m}$, particle radius $=0.25 \mu \mathrm{m}$ and surface fraction $=2 \%$. Microstructure reaching the stable state after $50 \mathrm{~s}(\mathrm{~b})$.

[10] G. Jinhua, G. Raymond, G. Thompson, Acta Metallurgica, 1997, 45, 3653-3658.

[11] M. Miodownik, Scripta Materialia, 2000, 42, 11731177 .

[12] L. Vanherpe, N. Moelans, B. Blanpain, S. Vandewalle, Computational Materials Science, 2010, 49, 340-350.

[13] K. Chang, W. Feng, L.-Q. Chen, Acta Materialia, 2009, 57, 5229-5236.

[14] D. Weygand, Acta Materialia, 1999, 47, 961-970.

[15] G. Couturier, C. Maurice, R. Fortunier, Philosophical Magazine, 2003, 83, 3387-3405.

[16] G. Couturier, R. Doherty, C. Maurice, R. Fortunier, Acta Materialia, 2005, 53, 977-989.

[17] K. Hitti, Ph.D. thesis, Ecole nationale supérieure des mines de Paris, 2011.

[18] N. Moelans, B. Blanpain, P. Wollants, Acta Materialia, 2006, 54, 1175-1184. 